\title{
As contribuiçóes da gameterapia no desempenho motor de indivíduo com paralisia cerebral ${ }^{1}$
}

\author{
Thiago da Silva Dias ${ }^{a}$, Karoline Faro da Conceiçăo ${ }^{a}$, Ana Irene Alves de Oliveira ${ }^{b}$, \\ Rafael Luiz Morais da Silva ${ }^{b}$ \\ aUniversidade Federal do Pará - UFPA, Belém, PA, Brasil.

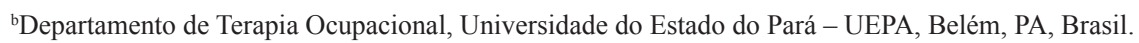

\begin{abstract}
Resumo: Objetivo: O estudo tem como objetivo analisar a utilização do Nintendo ${ }^{\circledR}$ Wii como recurso de (re) habilitação motora para indivíduos com paralisia cerebral (PC). Método: Esta é uma pesquisa de campo de caráter exploratório e quantitativo, na qual foi utilizado um estudo de caso. O sujeito foi avaliado e reavaliado quanto às habilidades motoras por meio do protocolo padronizado GMFM-88 (Gross Motor Function Measure-88) e foram realizadas dez sessões de gameterapia com o jogo Aladdin Magic Racer no período de fevereiro a março de 2013. Resultados: O sujeito obteve aumento percentual de 10,06\% no escore total da GMFM na reavaliação em relação ao obtido na avaliação inicial. Portanto, a análise dos dados denota uma mudança no desempenho motor do sujeito após a intervenção utilizando Nintendo ${ }^{\circledR}$ Wii. Conclusão: A gameterapia pode ser utilizada como um método de (re)habilitação motora para indivíduos com PC, bem como o Nintendo ${ }^{\circledR}$ Wii pode ser componente de um programa de (re)habilitação voltado a esse público, com foco na função motora grossa.
\end{abstract}

Palavras-chave: Paralisia Cerebral, Reabilitação, Tecnologia.

\section{The contributions of game therapy concerning motor performance of individual with cerebral palsy}

\begin{abstract}
Objective: In this study, it was analyzed the use of the videogame console Nintendo Wii ${ }^{\mathrm{TM}}$ as a motor (re) habilitation tool for individuals with Cerebral Palsy. Method: This is a field study with an exploratory and quantitative nature, in which is presented a case study. The participant's motor skills were assessed and reassessed using the standardized protocol GMFM-88 (Gross Motor Function Measure - 88). It were performed 10 game therapy sessions using the videogame "Aladdin Magic Racer" through February and March 2013. Results: The participant achieved a percentage increase of $10.06 \%$ in the total score of GMFM in the reassessment compared to the one achieved in the initial assessment. Thus, the data analysis showed that the participant's motor performance changed after the intervention using the Nintendo Wii ${ }^{\mathrm{TM}}$. Conclusion: Game therapy might be used as a motor (re)habilitation method for individuals with Cerebral Palsy, as well as Nintendo Wii ${ }^{\mathrm{TM}}$ might be a component of a (re)habilitation program for this population, focusing on gross motor function.
\end{abstract}

Keywords: Cerebral Palsy, Rehabilitation, Technology. 


\section{Introdução}

O desenvolvimento motor consiste em mudanças qualitativas e quantitativas das ações motoras do ser humano ao longo do tempo, em decorrência da interação entre as exigências da tarefa, os aspectos maturacionais do sujeito e as condiçóes ambientais. Essas mudanças possibilitam a realização de atividades diárias em padrôes de movimento que se caracterizam pela progressão da diversificação e da complexidade (GALLAHUE; OZMUN; GOODWAY, 2013; MASCARENHAS, 2008; SANTOS; DANTAS; OLIVEIRA, 2004).

$\mathrm{Na}$ infância, esse processo se constitui mediante a aquisição de um diversificado espectro de habilidades motoras que possibilitam à criança um amplo domínio do seu corpo em posturas estáticas e dinâmicas, locomover-se pelo meio ambiente de variadas formas e manipular objetos e instrumentos diversos (SANTOS; DANTAS; OLIVEIRA, 2004).

Entretanto, desordens neurológicas, como a paralisia cerebral (PC), podem impactar negativamente o desenvolvimento motor e, consequentemente, o desempenho funcional, o qual se refere ao desempenho dos sujeitos quanto a funções motoras, cognitivas, sociais, entre outras.

A PC é descrita como um grupo de distúrbios de movimento e postura com etiologias e quadros diversos, os quais causam limitaçóes na exploração do ambiente devido a uma lesão não progressiva do sistema nervoso central (SNC) em estágios precoces do desenvolvimento da criança (OZU; GALVÃO, 2005; ROSENBAUM et al., 2007; SANTOS; DANTAS; OLIVEIRA, 2004).

A lesão ocasiona diferentes níveis de déficits motores, os quais comprometem o desenvolvimento e a capacidade da criança de explorar e aprender ativamente no ambiente, visto que causam limitaçóes funcionais crônicas, as quais acarretam dificuldades nas atividades cotidianas (KOTT; HELD, 2003; MASCARENHAS, 2008; OZU; GALVÃO, 2005).

Neste sentido, o movimento voluntário da criança que, tipicamente, é complexo, coordenado e variado, torna-se descoordenado, estereotipado e limitado em razão do baixo controle seletivo da atividade dos grupos musculares (DIAS et al., 2010; KOTT; HELD, 2003; ROSENBAUM et al., 2007).

Desse modo, o sujeito com PC, quando comparado a indivíduos com desenvolvimento típico, desenvolve-se em um ritmo mais lento. Destaca-se que, independentemente do nível cognitivo, indivíduos com PC atingem os seus marcos motores mais tardiamente e muitos dos padrōes motores fundamentais, que deveriam emergir em certos estágios do desenvolvimento, podem estar ausentes (GALLAHUE, 2003; MADEIRA; CARVALHO, 2009).

Neste contexto, considerando-se as alteraçôes apresentadas e a dificuldade na manutenção de posturas, é fundamental para o indivíduo com $\mathrm{PC}$ a inclusão em programas de habilitação e reabilitação que viabilizem a sua participação em contextos relevantes (MONTEIRO et al., 2010).

Os programas terapêuticos pautados na promoção de aprendizagem motora têm o potencial de viabilizar tanto o processo de recuperaçáo de habilidades perdidas (reabilitaçáo) como o desenvolvimento de novas habilidades (habilitação). Portanto, doravante, esses dois processos serão agrupados sob o termo (re)habilitação.

Assim, destaca-se que um modo de minimizar as limitações advindas da PC é a viabilização de novas experiências ao sujeito, o que pode ser possibilitado pela utilização de recursos tecnológicos. Dentre esses recursos, há uma progressiva tendência no âmbito da (re)habilitação de indivíduos com PC a utilizar videogames (OLIVEIRA, 2010; MONTEIRO et al., 2011).

Os videogames surgiram no final da década de 1970 e configuram-se como uma modalidade de realidade virtual $(\mathrm{RV})$, visto que possibilitam ao indivíduo visualizar ambientes virtuais, manipular os elementos existentes no cenário e movimentar-se dentro do espaço. Os cenários são totalmente gerados por computador e, pelo fato de transportarem os elementos virtuais ao mundo real, viabilizam oportunidades de interaçôes singulares (CORRÊA et al., 2011).

Para Batista et al. (2012), videogames podem ser utilizados na (re)habilitaçáo de incapacidades físicas e/ou cognitivas, visando à viabilização de um ambiente motivador para a recuperação ou desenvolvimento de habilidades e capacidades perceptomotoras.

Neste sentido, essa modalidade de RV se caracteriza como uma forma de intervenção física, cognitiva ou psicológica que se baseia no uso de jogos e ambientes virtuais para viabilizar função a indivíduos com diferentes deficiências (CORRÊA et al., 2011).

Nesta perspectiva, destaca-se o Nintendo ${ }^{\circledR}$ Wii, cujo elemento diferencial é o Wii Remote ou Wiimote (controle remoto sem fio), o qual é conectado ao console via Bluetooth e possui três acelerômetros responsáveis por interpretar os movimentos tridimensionais nos eixos $\mathrm{x}$, y e z. Esse controle captura e reproduz na tela do jogo o movimento efetuado pelo indivíduo (CORREA et al., 2011; SAPOSNIK et al., 2010). 
Dessa forma, ao movimentar o controle, os movimentos do jogador são captados e transmitidos por uma barra de sensores, a qual triangula e infere posição e alinhamento. Assim, os movimentos físicos do usuário são refletidos na projeção, de um modo que os movimentos virtuais sejam semelhantes àqueles do plano material (CORRÊA et al., 2011).

Portanto, o Nintendo ${ }^{\circledR}$ Wii é de fácil aplicabilidade, promove a estimulação dos sistemas sensório-motor e cognitivo, bem como oferece um alto grau de motivação durante o tratamento (BATISTA et al., 2012).

Nesta perspectiva, esse recurso pode ser utilizado no contexto de tratamento das deficiências físicas e disfunções neuromotoras (incluindo a PC), visto que o feedback fornecido pela tela gera um reforço positivo, facilitando o treinamento e aperfeiçoamento de tarefas motoras (CORRÊA et al., 2011; SAPOSNIK et al., 2010).

Além disso, há estudos indicando que essa é uma área bastante promissora, tanto para utilização prática como para a realizaçáo de pesquisas que comprovem a eficácia da RV na melhora da funcionalidade de pessoas com diferentes deficiências (CORRÊA et al., 2011; SAPOSNIK et al., 2010). Especificamente, há evidências científicas que apoiam o uso de consoles de videogame, em conjunto com o tratamento convencional, como recursos terapêuticos na (re)habilitação de indivíduos com PC (SEGALA; OLIVEIRA; BRAZ, 2014).

Em um estudo desenvolvido por Monteiro et al. (2011), o jogo de boliche do Nintendo ${ }^{\circledR}$ Wii foi utilizado com um grupo de cinco crianças com PC para verificar o potencial da tarefa de promover aprendizagem motora. A análise dos dados indica que ocorreu aprendizagem, considerando-se a melhora de desempenho quanto aos escores obtidos - quantidade de pinos derrubados - pré- e pós-intervenção. Nesse estudo, os participantes tinham habilidades motoras compatíveis com os níveis II e III do Sistema de Classificação da Função Motora Grossa (GMFCS).

Em outro estudo realizado por Tavares et al. (2013) com duas crianças com PC, avaliadas pela GMFM-88 e pela Pediatric Balance Scale (PBS), o jogo Wii Fit do Nintendo ${ }^{\circledR}$ Wii foi utilizado em 20 sessôes, após as quais foram verificados aumentos nos escores totais da GMFM e da PBS. Esse resultado denotou a eficácia do recurso na melhora da função motora grossa e no equilíbrio de crianças com PC com comprometimento leve, ou seja, com habilidades motoras compatíveis com os níveis I e II do GMFCS.

Além destes, há outros estudos que abordam a utilização do Nintendo ${ }^{\circledR}$ Wii em crianças e adolescentes com PC, visando à melhora da função motora de membros superiores e inferiores, equilíbrio, controle postural e atividades de vida diária (SILVA et al., 2011; SILVA; IWABE-MARCHESE, 2015; TARAKCI et al., 2013; WINKELS et al., 2013).

Dessa forma, verifica-se que a utilização do Nintendo ${ }^{\circledR}$ Wii como recurso de (re)habilitação motora para indivíduos com PC é apoiada pela literatura científica, tanto quanto as bases teóricas relacionadas à $\mathrm{RV}$ como em relação a resultados de pesquisas práticas com esse grupo de indivíduos com base nesse recurso. Porém, ainda há escassez de estudos nacionais e, especificamente, na área de Terapia Ocupacional.

Neste estudo, o método no qual videogames são utilizados como recursos terapêuticos é denominado gameterapia. Assim, com base na literatura anteriormente citada e na necessidade de novos estudos, esta pesquisa tem o objetivo de analisar a utilização da gameterapia como um método de (re) habilitação motora para indivíduos com PC.

\section{Método}

\subsection{Aspectos éticos}

O estudo foi submetido à avaliação do Comitê de Ética em Pesquisa (CEP) envolvendo seres humanos da Universidade do Estado do Pará, em abril de 2014, tendo sido aprovado em junho do mesmo ano, emitindo o Certificado de Apresentação para Apreciaçáo Ética (CAAE) de nº 30678214.2.0000.5174 e o parecer n ${ }^{\circ} 673965$.

\subsection{Caracterização do estudo}

Esta é uma pesquisa de campo de caráter exploratório e quantitativo, na qual foi utilizado um estudo de caso.

\subsection{Ambiente}

A pesquisa foi realizada no Núcleo de Desenvolvimento em Tecnologia Assistiva e Acessibilidade (Nedeta) do Centro de Ciências Biológicas e da Saúde (CCBS) da Universidade do Estado do Pará (UEPA).

Nessa instituição, estudantes e profissionais de Terapia Ocupacional elaboram programas terapêuticos ocupacionais com base nos déficits funcionais e ocupacionais específicos de cada cliente, os quais são identificados por meio de instrumentos de avaliação padronizados e náo padronizados. Ressalta-se que 
a maior parte da clientela desse serviço possui disfunçóes neuromotoras graves, principalmente PC.

Este estudo aborda, especificamente, um programa de assistência baseado nos princípios da gameterapia, cujo enfoque foi a melhora de habilidades motoras.

\subsection{Participante}

O sujeito da pesquisa será referido pelo pseudônimo Yoshi neste estudo, a fim de preservar sua identidade. Ele apresenta PC do tipo atetoide, é do sexo masculino, tem 12 anos, cursa o quarto ano do Ensino Fundamental em uma instituição de ensino regular e frequenta regularmente o Nedeta. Yoshi também é atendido por outros profissionais das áreas de Terapia Ocupacional, Fisioterapia e Fonoaudiologia.

A funçâo motora do sujeito se caracteriza por movimentos involuntários lentos, suaves e atetóticos de membros superiores. Além disso, apresenta déficits de coordenação motora global e fina, com presença de dissinergia de grupos musculares opostos, o que afeta a execução de movimentos como flexão, extensão, pronação e supinação de membros superiores.

Ressalta-se que o sujeito deambula de maneira independente, apesar do déficit de equilíbrio dinâmico. Além disso, estabelece interação social de forma independente por meio de linguagem não verbal, visto que não consegue se comunicar de forma verbal.

Yoshi foi classificado como nível I quanto à função motora grossa, segundo os critérios do GMFCS - R\&E (revisado e expandido) para a faixa etária entre 12 e 18 anos. Essa classificação se deve ao fato de que Yoshi desempenha deambulação independente em diferentes contextos (casa, escola, Nedeta) e participa de diversas atividades cotidianas sem necessitar de dispositivos auxiliares para a mobilidade, o que o diferencia de um sujeito no nível II do GMFCS.

\subsection{Instrumentos e materiais}

\subsubsection{Gross Motor Function Measure-88 (GMFM-88)}

A GMFM ou medida da função motora grossa é um instrumento clínico destinado à avaliação da função motora grossa de sujeitos com PC. Possui duas versóes que se diferenciam pelo número de itens: a original, GMFM-88, e a mais recente, GMFM-66 (RUSSEL et al., 2011).
Esse instrumento foi validado para uso no contexto brasileiro e abrange um espectro de atividades correspondentes às habilidades de uma criança de 5 anos sem qualquer incapacidade motora, que envolvem deitar e rolar; sentar; engatinhar e ajoelhar; em pé; e andar, correr e pular (NUNES, 2008).

A aplicação requer que a criança demonstre as várias habilidades indicadas nas diretrizes e o sistema de pontuação é de quatro pontos: 0 : não consegue; 1: inicia; 2: parcial; 3: total. Na GMFM-88, o cálculo consiste na soma da pontuação dos diferentes itens para obtenção de uma pontuação bruta ou percentual em cada uma das cinco dimensóes, as áreas-meta e o total (RUSSEL et al., 2011).

\subsubsection{Gross Motor Function Classification System (GMFCS)}

O GMFCS ou sistema de classificação da função motora grossa é um sistema de cinco níveis utilizado para classificar a funçáo motora grossa de sujeitos com PC. É confrável para ser utilizado no Brasil, por profissionais de saúde com diferentes níveis de experiência (PALISANO et al., 2007; SILVA, 2013).

De modo geral, a distinção entre os níveis é a seguinte: I: anda sem limitaçóes; II: anda com limitaçôes; III: anda utilizando um dispositivo manual de mobilidade; IV: automobilidade com limitaçóes, a qual pode ser mobilidade motorizada; V: transportado em cadeira de rodas manual (PALISANO et al., 2007).

A versão expandida e revisada do sistema pode ser utilizada com crianças e adolescentes até a idade de 18 anos, de modo que há descrições de habilidades e limitaçôes funcionais específicas para as seguintes faixas etárias: menos de 2 anos (considerando a idade corrigida), entre 2 e 4 anos, entre 4 e 6 anos, entre 6 e 12 anos e entre 12 e 18 anos (PALISANO et al., 2007).

\subsection{Etapas da pesquisa}

\subsubsection{Seleção do sujeito}

Os critérios de inclusão da pesquisa foram: sujeito com diagnóstico de PC, com idade entre 10 e 18 anos; função motora de níveis I a III no GMFCS, visto que nos níveis IV e $\mathrm{V}$ os sujeitos podem apresentar déficits no controle de tronco e cervical, os quais podem interferir na manutenção da coordenação viso-motora durante a execução da tarefa; estabelecer comunicação efetiva com os pesquisadores; não apresentar doenças cardiorrespiratórias; atendido regularmente no Nedeta; concordar com o termo de assentimento livre e esclarecido; responsáveis legais 
devem aceitar as cláusulas propostas no termo de consentimento livre e esclarecido.

Em contrapartida, foram excluídos os sujeitos que apresentaram outra patologia, deficiência intelectual diagnosticada e com baixa assiduidade ao Nedeta e, além disso, que não estabelecessem comunicação efetiva com os pesquisadores, com função motora acima do nível III no GMFCS, que apresentassem doenças cardiorrespiratórias e possuíssem quadros convulsivos.

O participante da pesquisa foi selecionado com base em técnica de amostragem não aleatória, na qual o sujeito atendeu aos critérios de inclusão, mostrou disponibilidade de horário compatível com a dos pesquisadores e demonstrou interesse na utilização do console como método de (re)habilitação.

\subsubsection{Avaliação pré-intervenção}

Inicialmente, o sujeito foi avaliado quanto às habilidades motoras por meio do protocolo padronizado GMFM-88.

Este tem sido o instrumento de avaliação quantitativo mais indicado para ser usado na detecção e mensuração de mudanças na função motora grosseira em sujeitos com PC. Porém, configura um sistema para quantificar a função motora e não para saber como o sujeito desempenha essa funçáo, ou seja, caracteriza o seu nível de função sem considerar a qualidade do desempenho (FERNANDES, 2009).

\subsubsection{Intervenções}

Posteriormente, foram realizadas dez sessões de gameterapia com o sujeito, nas quais Nintendo ${ }^{\circledR}$ Wii foi utilizado como recurso terapêutico em sessóes de aproximadamente 40 minutos, duas vezes por semana, no período de fevereiro a março de 2013.

$\mathrm{O}$ protocolo de pesquisa operacionalizado foi adaptado do estudo de Tavares et al. (2013). O número e a frequência das sessóes foram arbitrariamente definidos conforme a dinâmica de atendimentos no Nedeta.

Especificamente, utilizou-se o Aladdin Magic Racer, um jogo de corrida no qual são utilizados tapetes voadores como veículos, cujo diferencial é o modo de jogabilidade: movimentaçáo bimanual do controle sem necessidade de apertar botóes. Desse modo, a tarefa é simplificada e torna-se mais acessível a sujeitos com deficiência motora, pois dispensa a destreza manual para realizar a movimentação direita-esquerda por meio de botóes, geralmente necessária para comandar o veículo em jogos de corrida típicos.

Destaca-se que, no ambiente do jogo, há outros quatro jogadores virtuais e o sujeito necessita desviar de obstáculos durante o percurso. Além disso, há recursos que podem atribuir mais velocidade ao personagem durante o jogo.

Com base nessas variáveis do jogo, é possível identificar melhora no desempenho do sujeito segundo os parâmetros "média de tempo" e "melhor colocação", visto que estão diretamente relacionados a mudanças nas habilidades motoras do jogador subjacentes à melhora nas capacidades de ultrapassar os adversários, desviar de obstáculos e utilizar os recursos que lhe conferem mais velocidade.

\subsubsection{Avaliação pós-intervenção}

Ao término das intervenções, foi realizada a reavaliação utilizando GMFM para verificar o desempenho do indivíduo após intervenção terapêutica ocupacional, mediante o uso da gameterapia, e analisar as alteraçôes nos escores pré- e pós-intervenção.

\subsubsection{Análise dos resultados}

A análise dos dados da pesquisa ocorreu por meio da quantificaçáo do desempenho do sujeito durante as sessóes de gameterapia e comparação entre os escores obtidos na avaliação e na reavaliação com a GMFM, a fim de identificar o desempenho motor do sujeito diante da intervenção realizada.

\section{Resultados e Discussão}

\subsection{Avaliação pré-intervenção}

$\mathrm{Na}$ avaliação inicial com GMFM, o sujeito apresentou dificuldades em todas as dimensões do protocolo. Isto pode ser atribuído à atetose e ao déficit de coordenação motora grossa, os quais dificultam o desempenho de tarefas motoras que envolvam a ação coordenada dos grupos musculares.

Apesar disso, segundo informações da mãe, Yoshi consegue ser independente nas atividades cotidianas, embora demande mais tempo para realizar as tarefas, principalmente aquelas de coordenaçóes bimanual e visomotora.

A partir do perfil motor traçado pela GMFM, foram realizadas intervençôes visando promover a melhora da função motora, principalmente de membros superiores. O jogo escolhido foi Aladdin Magic Racer, visto que viabiliza uma tarefa que exige 
movimentos integrados de membros superiores e coordenação olho-mão para desempenhar as açóes requeridas pelo jogo.

\subsection{Desempenho durante a intervenção}

O desempenho de Yoshi durante as sessōes de gameterapia é apresentado na Tabela 1 e na Figura 1, as quais demonstram que o sujeito conseguiu diminuir, de modo não linear, o tempo médio de execução da tarefa ao longo dos atendimentos. Isto denota a fase de aquisição no processo de aprendizagem motora, na qual o sujeito, progressivamente, desenvolve padróes adaptativos de movimentação, os quais acarretam melhores resultados no contexto do jogo.

Esse processo de aquisição da aprendizagem motora pode ser inferido por meio do desempenho, enquanto sua melhora pode ser observada pelo aumento da consistência, fluência no movimento, diminuiçáo do erro de execução e redução no tempo total de movimento para a realização da tarefa (MONTEIRO et al., 2011).

Tabela 1. Pontuação no jogo Aladdin Magic Racer.

\begin{tabular}{|c|c|c|}
\hline & Média de tempo & Melhor colocação \\
\hline 1 & 02 '23” 29 & $5^{\circ}$ \\
\hline 2 & $02^{\prime} 23^{\prime \prime} 92$ & $4^{\circ}$ \\
\hline 3 & $02 ’ 25 ” 40$ & $5^{\circ}$ \\
\hline 4 & $02 ’ 25 ” 08$ & $5^{\circ}$ \\
\hline 5 & $02 ’ 22 ” 93$ & $5^{\circ}$ \\
\hline 6 & 02 ' 19” 30 & $1^{\mathrm{o}}$ \\
\hline 7 & 02 '24” 01 & $5^{\circ}$ \\
\hline 8 & $02^{\prime} 20^{\prime \prime} 86$ & $4^{\circ}$ \\
\hline 9 & $02^{\prime} 17^{\prime \prime} 20$ & $3^{\circ}$ \\
\hline 10 & 02 ' 17" 66 & $2^{\circ}$ \\
\hline
\end{tabular}

Legenda: média de tempo em ' (minutos), " (segundos) e décimos. Fonte: pesquisa de campo, 2013.
Conforme mencionado na metodologia, os parâmetros "média de tempo" e "melhor colocação" denotam a progressiva melhora na habilidade do sujeito de desempenhar o jogo de modo mais eficiente. Esses parâmetros estão diretamente relacionados ao processo de aquisição das habilidades de ultrapassar os adversários, desviar de obstáculos e utilizar os recursos do jogo que the conferem mais velocidade.

Assim, a diminuição do tempo se torna importante como um dado que demonstra o desenvolvimento de habilidade motora por meio da prática ao longo das sessôes, a qual influenciou no tempo e tornou a tarefa mais fácil de ser desempenhada.

O desenvolvimento desses padrôes motores ocorre por um processo interno que produz alteraçóes consistentes no comportamento individual em decorrência da interação, da experiência, da educação e/ou do treinamento com processos biológicos (MONTEIRO; PASIN, 2011).

Desse modo, a experiência de interação com o Nintendo ${ }^{\circledR}$ Wii mobilizou a ocorrência desse processo interno decorrente da interação entre a função motora previamente mensurada de Yoshi com as exigências do jogo, o que acarretou a aprendizagem de novos padrôes de movimentação. Destaca-se que a falta de linearidade quanto ao fator tempo durante o desempenho do jogo remete à noção de que a aquisiçáo da habilidade motora não segue um padrão linear.

Nesse sentido, o desenvolvimento motor é compreendido, em uma perspectiva dinâmica, como um processo descontínuo, no qual a mudança individual ao longo do tempo não é necessariamente tranquila e hierárquica, bem como não precisa envolver uma mudança em direção a níveis mais elevados de complexidade e competência no sistema motor, pois o desenvolvimento, apesar de, por definição, ser um processo contínuo, não é necessariamente linear (GALLAHUE; OZMUN; GOODWAY, 2013).

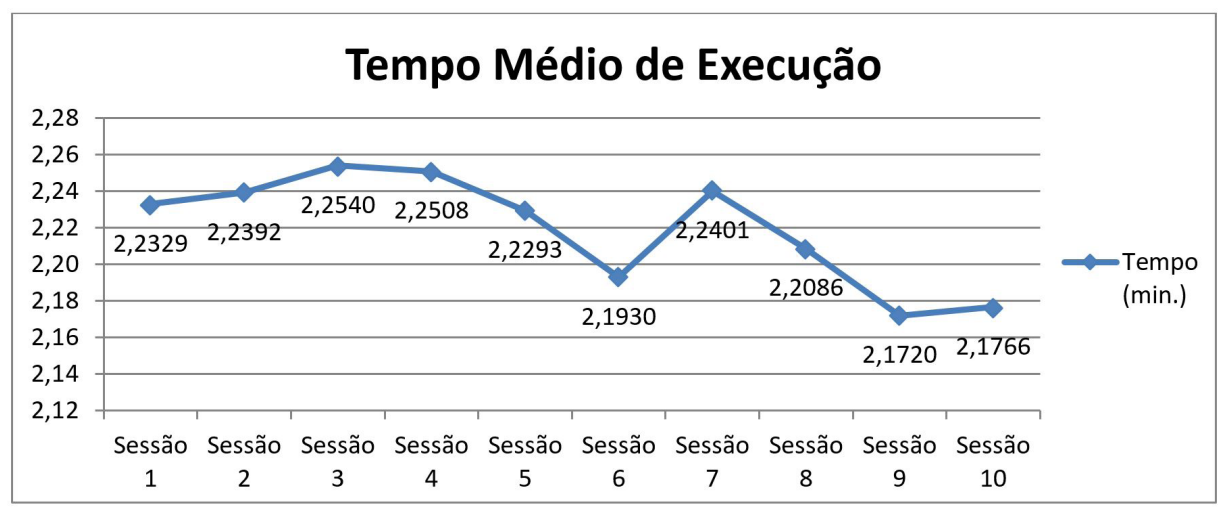

Figura 1. Variação do tempo médio de execução ao longo das sessões. Fonte: pesquisa de campo, 2013. 


\subsection{Análise pós-intervenção}

As tarefas motoras realizadas por meio do jogo Aladdin Magic Racer, apesar de não levarem o indivíduo a desenvolver padróes complexos de movimento, viabilizaram a emergência de padróes de função motora grossa subjacentes a atividades envolvendo a coordenaçáo de grupos musculares (principalmente de punho e cotovelo). Esses padróes foram representados nas sessôes pela utilização do controle sem fio para desempenhar as tarefas do jogo anteriormente citado.

Essa afirmação pode ser verificada pela avaliação realizada após a intervenção. A Tabela 2 e a Figura 2 mostram a comparação entre os escores apresentados por Yoshi na GMFM pré- e pós-intervenção.

Esses dados ratificam a perspectiva de que o desempenho do jogo Aladdin Magic Racer ao longo de dez sessóes viabilizou o desenvolvimento de padróes motores mais adaptados ao sujeito deste estudo. Quanto a isso, destaca-se que foi verificado aumento nos escores de praticamente todas as dimensóes do protocolo, exceto na $\mathrm{D}$ (de pé), na qual Yoshi obteve o mesmo escore.

Em detrimento disso, as dimensões $\mathrm{A}, \mathrm{B}, \mathrm{C}$ e $\mathrm{E}$ apresentaram aumento, ainda que discreto, nos escores individuais, o que é atribuído à prática sucessiva da coordenação de movimentos, a fim de obter um padrão mais adaptado de função motora grossa. Assim, conforme apresentado na Tabela 2, houve aumento percentual de $10,06 \%$ no escore total na GMFM entre avaliação $(77,64 \%)$ e reavaliação (87,70\%).

Além disso, houve aumento percentual de $11,76 \%$ no escore da reavaliação $(94,11 \%)$ em relação ao obtido pelo sujeito na avaliação inicial $(82,35 \%)$ na dimensão A, que se refere a tarefas realizadas na posição deitado. Esse resultado pode ser atribuído à melhora na integração bilateral viabilizada pelo modo de jogabilidade do Nintendo ${ }^{\circledR}$ Wii. Essa habilidade favorece a realização de movimentos de ambos os membros para o desempenho de tarefas que exigem mudança de decúbito componentes dessa dimensão da GMFM.

Tabela 2. Escores na avaliação do sujeito após a intervenção.

\begin{tabular}{|c|c|c|c|}
\hline Dimensão & $\begin{array}{c}\text { Pontuação } \\
\text { pré-intervenção (\%) }\end{array}$ & $\begin{array}{c}\text { Pontuação } \\
\text { pós-intervenção }(\%)\end{array}$ & $\begin{array}{c}\text { Progressão } \\
\text { percentual (\%) }\end{array}$ \\
\hline A - Deitando e rolando & 82,35 & 94,11 & 11,76 \\
\hline B - Sentando & 91,66 & 93,33 & 1,67 \\
\hline C - Engatinhando e ajoelhando & 85,71 & 92,85 & 7,14 \\
\hline D - Em pé & 74,35 & 74,35 & 0 \\
\hline E - Andando, correndo e pulando & 54,16 & 63,88 & 9,72 \\
\hline Total & 77,64 & 87,70 & 10,06 \\
\hline
\end{tabular}

Fonte: pesquisa de campo, 2013.

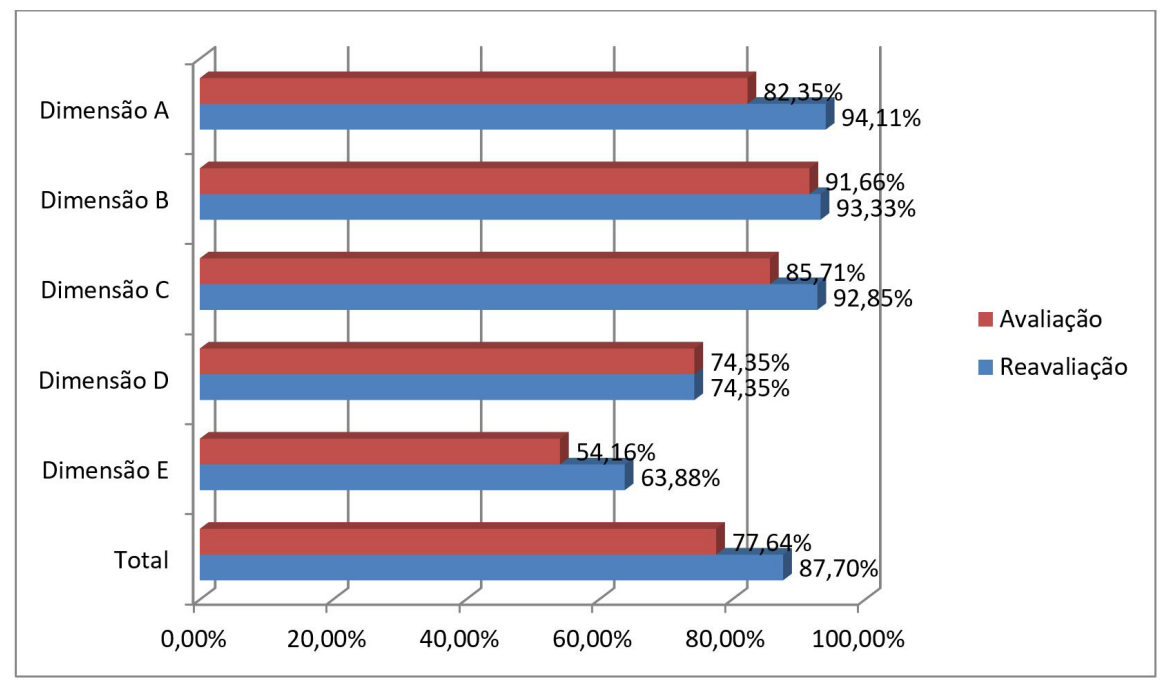

Figura 2. Variação dos escores por dimensão da GMFM. Fonte: pesquisa de campo, 2013. 
Quanto à dimensão $\mathrm{B}$, que se refere a tarefas realizadas na posição sentado, verifica-se que houve aumento percentual de $1,67 \%$ entre avaliação (91,66\%) e reavaliaçáo $(93,33 \%)$, o que pode ser atribuído ao desempenho do jogo na posição sentado e à estimulação do sujeito quanto à manutenção da postura durante as sessóes. A melhora no desempenho de atividades na posição sentado acarretou discreta progressão no desempenho das tarefas inerentes a essa dimensão.

$\mathrm{Na}$ dimensão C (tarefas realizadas nas posiçóes de quatro e ajoelhado), por sua vez, houve aumento percentual de $7,14 \%$ entre avaliação $(85,71 \%)$ e reavaliação $(92,85 \%)$. Isto também pode ser atribuído à melhora da integraçáo bilateral decorrente do desempenho do jogo Aladdin Magic Racer e acarretou, principalmente, melhor desempenho da tarefa de engatinhar, que exige movimentação coordenada dos membros e compóe o escopo de tarefas dessa dimensão.

O sujeito não apresentou evolução de escore na dimensão $\mathrm{D}$ (tarefas na posição em pé), que se manteve estável no percentual de 74,35\% entre avaliação e reavaliação, o que pode ser atribuído à dificuldade do sujeito de manter uma posição estática devido à atetose.

Por fim, na dimensão E (tarefas realizadas em posições dinâmicas, com mudança da base de equilíbrio), houve aumento percentual de $9,72 \%$ entre avaliação $(54,16 \%)$ e reavaliação $(63,88 \%)$, o que pode ser atribuído, novamente, à melhora na integraçáo bilateral, a qual possibilita ao sujeito melhorar a habilidade de utilizar reaçóes de equilíbrio necessárias para desempenhar as tarefas dessa dimensão.

Assim, a análise dos dados denota que houve uma mudança no desempenho motor do sujeito após a intervenção utilizando Nintendo ${ }^{\circledR}$ Wii. Isto é apoiado pelo aumento da maioria dos escores da GMFM, desde as tarefas mais simples na posição sentado até aquelas de caráter mais complexo em posiçôes de mudança dinâmica da base de apoio, como correr e pular. Nesse sentido, esse recurso pode ser utilizado no contexto de tratamento das deficiências físicas e disfunçôes neuromotoras (CORRÊA et al., 2011).

\section{Conclusão}

Verifica-se que as tarefas motoras inerentes ao videogame estimulam o aprimoramento da função motora grossa. Desse modo, a gameterapia, especificamente por meio do jogo Aladdin Magic
Racer, pode ser sugerida como um método de (re) habilitação motora para sujeitos com PC.

Assim, o Nintendo ${ }^{\circledR}$ Wii é um recurso que pode ser componente de um programa de (re)habilitação voltado a esse público, com foco na funçáo motora grossa, pois apresentou resultados satisfatórios, apoiados por um protocolo de avaliação padronizado.

Além disso, o desenvolvimento da função motora grossa, com o jogo de videogame, pode viabilizar um melhor desempenho em atividades da vida cotidiana que exijam coordenação motora grossa. Nesse sentido, por exemplo, o sujeito que aprimora a coordenação dos grupos musculares do ombro e do cotovelo, por meio das sessóes de gameterapia, pode, consequentemente, adquirir um melhor desempenho na escrita, alimentação, pintura, escovação dos dentes, entre outras atividades que requerem um nível adequado de coordenação motora para serem desenvolvidas.

\section{Limitações}

Nesta pesquisa, não foi possível utilizar um modelo experimental com um número representativo de participantes e/ou utilização de um grupo controle, em razáo de fatores como limitação de tempo para a execução da pesquisa, baixa assiduidade de alguns participantes, dificuldades com a obtençáo de locais adequados para as intervençóes, entre outros.

O uso de um estudo de caso representa uma limitação quanto ao controle de variáveis e validade externa, o que dificulta a generalizaçáo dos resultados para outros sujeitos com PC com graus de habilidade motora superiores a I no GMFCS e/ou com PC do tipo espástico e atáxico. Porém, utilizou-se este estudo de caso objetivando apresentar uma abordagem quanto ao uso de videogames como recurso de (re) habilitação motora por terapeutas ocupacionais e suscitar a replicação e/ou modificação da metodologia em pesquisas futuras, incluindo a utilização de grupos-controle.

\section{Referências}

BATISTA, J. S. et al. Reabilitaçáo de idosos com alteraçôes cognitivas através do Nintendo ${ }^{\circledR}$ Wii. Revista Brasileira de Ciências do Envelhecimento Humano, Passo Fundo, v. 9, n. 2, p. 293-299, 2012.

CORRÊA, A. G. D. et al. Realidade virtual e jogos eletrônicos: uma proposta para deficientes. In: MONTEIRO, C. B. M. (Org.). Realidade virtual na Paralisia Cerebral. São Paulo: Plêiade, 2011. p. 93-108.

DIAS, A. C. B. et al. Desempenho funcional de crianças com Paralisia Cerebral participantes de tratamento mul- 
tidisciplinar. Fisioterapia e Pesquisa, São Paulo, v. 17, n. 3, p. 225-229, 2010.

FERNANDES, M. V. Comparação entre as técnicas de tratamento com exercicio de resistência progressiva e conceito neuroevolutivo bobath no torque muscular e na função motora grosseira em crianças portadoras de diplegia espástica. 2009. 87 f. Dissertaçáo (Mestrado em Educação Física) - Universidade São Judas Tadeu, São Paulo, 2009.

GALLAHUE, D. L. Compreendendo o desenvolvimento motor: bebês, crianças, adolescentes e adultos. São Paulo: Phorte Editora, 2003.

GALlAHUE, D. L.; OZMUN, J. C.; GOODWAY, J. D. Compreendendo o desenvolvimento motor: bebês, crianças, adolescentes e adultos. Porto Alegre: AMIGH, 2013.

KOTT, K. M.; HELD, S. L. Effects of orthoses on upright functional skills of children and adolescents with Cerebral Palsy. Pediatric Physical Therapy, Baltimore, v. 14, n. 4, p. 199-207, 2003.

MADEIRA, E. A. A.; CARVALHO, S. G. Paralisia Cerebral e fatores de risco ao desenvolvimento motor: uma revisão teórica. Cadernos de Pós-Graduação em Distúrbios do Desenvolvimento, São Paulo, v. 9, n. 1, p. 142-163, 2009.

MASCARENHAS, T. Análise das escalas desenvolvidas para avaliar a função motora de pacientes com Paralisia Cerebral. 2008. 65 f. Dissertação (Mestrado em Ciências da Saúde) - Santa Casa, São Paulo, 2008.

MONTEIRO, C. B. M. et al. Aprendizagem motora em crianças com Paralisia Cerebral. Revista Brasileira de Crescimento e Desenvolvimento Humano, São Paulo, v. 20, n. 2, p. 250-262, 2010.

MONTEIRO, C. B. M. et al. Paralisia Cerebral e aprendizagem de jogo eletrônico (Nintendo Wii). In: MONTEIRO, C. B. M. (Org.). Realidade virtual na Paralisia Cerebral. São Paulo: Plêiade, 2011. p. 111-142.

MONTEIRO, C. B. M.; PASIN, C. T. Aprendizagem motora: um elo entre deficiência e realidade virtual. In: MONTEIRO, C. B. M. (Org.). Realidade virtual na Paralisia Cerebral. São Paulo: Plêiade, 2011. p. 93-108.

NUNES, L. C. B. G. Tradução e validação de instrumentos de avaliaçâo motora e de qualidade de vida em Paralisia Cerebral. 2008. 245 f. Tese (Doutorado em Engenharia Elétrica) - Universidade Estadual de Campinas, Campinas, 2008.

OLIVEIRA, A. I. A. Integrando tecnologias para leitura em crianças com Paralisia Cerebral na educaçâo inclusiva. 2010. 145 f. Tese (Doutorado em Teoria e Pesquisa do Comportamento) - Universidade Federal do Pará, Belém, 2010.

OZU, M. H. U.; GALVÃO, M. C. S. Fisioterapia na Paralisia Cerebral. In: MOURA, E. W.; SILVA, P. A. C. Fisioterapia: aspectos clínicos e práticos da reabilitação. São Paulo: Artes Médicas, 2005. p. 27-50.
PALISANO, R. et al. GMFCS $-E \& R$ : Gross Motor Function Classification System expanded and revised. Hamilton: CanChild Centre for Childhood Disability Research, 2007. Disponível em: <https://www.cpqcc. org/sites/default/files/documents/HRIF_QCI_Docs/ GMFCS-ER.pdf>. Acesso em: 8 abr. 2014.

ROSENBAUM, P. et al. A report: the definition and classification of cerebral palsy. Developmental Medicine \& Child Neurology, London, v. 49, n. 6, p. 8-14, 2007. Suplemento.

RUSSEL, D. J. et al. Medida da Função Motora Grossa [GMFM-66 \& GMFM-88]: manual do usuário. São Paulo: Memnon, 2011.

SANTOS, S.; DANTAS, L.; OLIVEIRA, J. A. Desenvolvimento motor de crianças, de idosos e de pessoas com transtorno da coordenação. Revista Paulista de Educação Física, São Paulo, v. 18, p. 33-44, 2004. Número Especial.

SAPOSNIK, G. et al. Effectiveness of virtual reality using Wii gaming technology in stroke rehabilitation: a pilot randomized clinical trial and proof of principle. Stroke, Baltimore, v. 41, n. 7, p. 1477-1484, 2010.

SEGALA, M.; OLIVEIRA, G. C.; BRAZ, M. M. Utilização do Nintendo ${ }^{\circledR}$ Wii como recurso terapêutico no tratamento da Paralisia Cerebral: uma revisão integrativa. Saúde, Santa Maria, v. 40, n. 1, p. 17-22, 2014.

SILVA, D. B. R. Classificação da Função Motora Grossa e habilidade manual de crianças com Paralisia Cerebral: diferentes perspectivas entre pais e terapeutas. 2013. 164 f. Tese (Doutorado em Ciências) - Universidade de São Paulo, Ribeirão Preto, 2013.

SILVA, M. Z. et al. Efetividade da gameterapia no controle postural de uma criança com paralisia cerebral hemiplégica espastica. In: CONGRESSO BRASILEIRO MULTIDISCIPLINAR DE EDUCAÇÃO ESPECIAL, 6., 2011, Londrina. Anais... Londrina, 2011. p. $3094-$ 3106.

SILVA, R. R.; IWABE-MARCHESE, C. Using virtual reality for motor rehabilitation in a child with Ataxic Cerebral Palsy: case report. Fisioterapia e Pesquisa, São Paulo, v. 22, n. 1, p. 97-102, 2015.

TARAKCI, D. et al. Wii-based balance therapy to improve balance function of children with Cerebral Palsy: a pilot study. Journal of Physical Therapy Science, Tóquio, v. 25, n. 9, p. 1123-1127, 2013.

TAVARES, C. N. et al. Uso do Nintendo ${ }^{\circledR}$ Wii para reabilitação de crianças com paralisia cerebral: estudo de caso. Revista Neurociências, São Paulo, v. 21, n. 2, p. 286293, 2013.

WINKELS, D. G. M. et al. Wii ${ }^{\text {TM}}$-habilitation of upper extremity function in children with Cerebral Palsy. an explorative study. Developmental Neurorehabilitation, Londres, v. 16, n. 1, p. 44-51, 2013. 


\section{Contribuição de Autores}

Os autores Thiago da Silva Dias e Karoline Faro da Conceição são responsáveis pela concepção, pesquisa bibliográfica, coleta de dados, análise, interpretação e discussão dos resultados, assim como redação final do artigo. Os autores Rafael Luiz Morais da Silva e Ana Irene Alves de Oliveira realizaram orientação teórica e metodológica do estudo e auxiliaram na análise e interpretação dos resultados. Todos os autores aprovaram a versão final do texto.

\section{Nota}

${ }^{1} \mathrm{O}$ estudo é integrante do projeto de pesquisa intitulado "O Nintendo Wii como recurso terapêutico na (re)habilitação cognitiva e motora de indivíduos com paralisia cerebral”, o qual foi aprovado no edital 052/2012 do Programa Institucional de Bolsas de Iniciação Científica (Pibic), financiado pelo Conselho Nacional de Desenvolvimento Científico e Tecnológico (CNPq). Todos os procedimentos éticos para a realização da pesquisa foram realizados, aprovados pelo Comitê de Ética em Pesquisa em Seres Humanos do Centro de Ciências Biológicas e da Saúde da Universidade do Estado do Pará (Certificado de Apresentaçáo para Apreciação Ética [CAAE] no 30678214.2.0000.5174 e parecer no 673965). Um resumo deste artigo foi apresentado em forma de pôster no Neurological Disorders Summit (NDS-2015), São Francisco - Estados Unidos, o qual ocorreu no período de 06 a 08 de Julho de 2015. Este é um artigo original e inédito, o qual não está sendo avaliado para publicação em outra revista e, exceto pela publicação como resumo no evento supracitado, ainda não foi parcial ou integralmente publicado. 